\section{PRODUCCIÓN DE SEMILLA DE CRUZAS SIMPLES DE HÍBRIDOS DE MAÍZ EN TLAXCALA, MÉXICO}

\section{SEED PRODUCTION OF SINGLE CROSSES OF MAIZE HYBRIDS IN TLAXCALA, MÉXICO}

\author{
Juan Virgen Vargas ${ }^{1}$, José L. Arellano Vázquez ${ }^{1}$, \\ Israel Rojas Martínez ${ }^{2}$, Miguel A. Ávila Perches ${ }^{3}$ \\ y Germán F. Gutiérrez Hernández ${ }^{4}$
}

${ }^{1}$ Campo Experimental Valle de México, Centro de Investigciones Regional del Centro (CIRCE). Instituto Nacional de Investigaciones Forestales, Agrícolas y Pecuarias, INIFAP. Km 18.5 Carretera Los Reyes-Lechería. 56230, Chapingo, Edo. de México. Tel. 01(595) 9542877 Ext. 137. ${ }^{2}$ Sitio Experimental Tlaxcala. CIRCE. INIFAP. Km. 2.5 Carr. Tlaxcala-Chiautempan. 90800. Chiautempan, Tlaxcala. ${ }^{3}$ Campo experimental Bajío, CIRCE, INIFAP. Km. 6.5 Carr. CelayaSan Miguel de Allende. 38110, Celaya, Guanajuato. ${ }^{4}$ Bioprocesos, Unidad Interdisciplinaria de Biotecnología (UPIBI), Instituto Politécnico Nacional. Av. Acueducto s/n. La Laguna Ticomán. 07340, México, D. F.

*Autor para correspondencia (jvirgen_vargas@hotmail.com)

\section{RESUMEN}

El Instituto Nacional de Investigaciones Forestales, Agrícolas y Pecuarias (INIFAP) de México produce semilla para atender la demanda de los productores de semilla certificada de híbridos de maíz (Zea mays L.), y desarrolla tecnología para identificar la localidad y el manejo agronómico óptimos para producirla. En el año 2008 se evaluó el rendimiento y la calidad física de semilla de siete cruzas simples progenitoras femeninas de híbridos comerciales de maíz, en dos densidades de población (62 y 83 mil plantas ha ${ }^{-1}$ ), en dos localidades del Estado de Tlaxcala (Espíritu Santo, 2480 msnm; Francisco Villa, 2540 msnm). En cada localidad se utilizó el diseño de bloques completos al azar con tres repeticiones. El análisis combinado se hizo con arreglo factorial de las siete cruzas simples y las dos densidades de población. Hubo diferencias significativas $(\mathbf{P} \leq$ $0.05)$ entre localidades, cruzas simples $y$ en la interacción localidad $x$ densidad de población para rendimiento y tamaño de semilla, y entre densidades de población para tamaño de semilla. El rendimiento de semilla de las cruzas simples de los híbridos 'H-64', 'H-66' y 'H-68' (7.0, 6.4 y $\left.6.2 \mathrm{t} \mathrm{ha}^{-1}\right)$ superó $(\mathrm{P} \leq 0.01)$ al de la cruza simple del 'H-50' (4.4 t ha' $\left.{ }^{-1}\right)$. No hubo diferencias $(P \leq 0.05)$ entre densidades para rendimiento, aunque a 83 mil plantas ha ${ }^{-1}$ la semilla fue de menor tamaño y peso que a la densidad de 62 mil plantas ha $^{-1}$. El alto rendimiento y buena calidad física de la semilla de las cruzas simples de los híbridos 'H-68', 'H-64' y 'H-66' indican que es factible producir su semilla en estas dos localidades del Edo. de Tlaxcala.
Palabras clave: Zea mays, densidad de población, rendimiento, tamaño de semilla.

\section{SUMMARY}

The Instituto Nacional de Investigaciones Forestales, Agrícolas y Pecuarias (INIFAP) of México produces seed to attend the demand of certified seed producers of maize hybrids (Zea mays $\mathbf{L}$.), and also develops technology for the identification of the optimal location for seed production and agronomic management. In this study yield and physical quality of seed of female single crosses of commercial maize hybrids were evaluated in two population densities $(63000$ and 83 000 plants ha $^{-1}$ ) at two localities of Tlaxcala State (Espíritu Santo, 2480 masl; Francisco Villa, 2540 masl). A randomized complete blocks design with three replications was used in each location, and a combined statistical analysis with factorial arrangement of seven single crosses and two plant densities was applied. We found significant differences $(P \leq \mathbf{0 . 0 5})$ among locations, single crosses and location $\mathrm{x}$ population density interaction for seed yield and seed size. Differences were also present between plant densities for seed size. Seed yield of the single crosses of hybrids 'H-64', 'H-66' and 'H-68' (7.0, 6.4 and 6.2 $\left.\mathrm{t} \mathrm{ha}^{-1}\right)$ was higher $(\mathrm{P} \leq 0.01)$ that yield of the single cross of 'H-50' $\left(4.4 \mathbf{t ~ h a}^{-1}\right)$. No differences $(\mathrm{P} \leq \mathbf{0 . 0 5})$ were detected between plant densities for seed yield, although at 83000 plants ha ${ }^{-1}$ seeds were smaller in size and weight, compared to the density of 62 000 plants ha $^{-1}$. The high yield and good physical quality of seeds of single crosses from maize hybrids ' $\mathrm{H}-64$ ', 'H-66' and 'H-68' shows that it is feasible to produce their seeds in these two localities of Tlaxcala State.

Index words: Zea mays, population density, yield, seed size.

\section{INTRODUCCIÓN}

El Instituto Nacional de Investigaciones Forestales Agrícolas y Pecuarias (INIFAP) participa en la producción de semilla para atender la demanda de semilla certificada, y desarrolla tecnología para optimizar la produccion. Actualmente son las empresas semilleras de capital transnacional las que comercializan la mayor cantidad de semilla certificada de maíz (Zea mays L.) en México. Ante esta situación el INIFAP en Tlaxcala promueve el fortalecimiento de empresas de capital nacional a través de la transferencia de nuevos híbridos y variedades mejoradas de maíz y de la tecnología de producción de semilla, para reducir los costos de semilla certificada y así beneficiar a las empresas semilleras nacionales y a los productores de grano.

En el Estado de Tlaxcala el maíz de temporal o secano ocupa una superficie aproximada anual de 120 mil hectáreas, que equivalen a $60 \%$ de la superficie total cultivada en el estado, con un rendimiento medio de 2.76 t ha ${ }^{-1}$ y una producción estatal de 315392 t anuales. La baja productividad del maíz en la entidad se atribuye en parte a que $90 \%$ de la superficie se siembra con variedades criollas de baja capacidad de respuesta a los fertilizantes y a las altas densidades de población (SIAP, 2009). En el estado se siembran 64028 ha con híbridos de maíz en condiciones de punta de riego y en temporal 
de muy buena y buena productividad, que representan los ambientes con mayor potencial productivo para producir semilla de maíz, donde es posible lograr rendimientos de 8 t ha ${ }^{-1}$ (María-Ramírez y Rojas-Martínez, 2003).

Según Beck (2002), para producir semilla de maíz es esencial seleccionar un ambiente de producción con abundante luminosidad durante la estación de crecimiento y con suficiente lluvia pero no excesiva, y bien distribuida, particularmente durante el periodo de floración. La información acerca de la importancia de la densidad de población en el rendimiento de grano y características agronómicas del cultivo es abundante, pero escasa en cuanto a la producción de semilla. En el Estado de Tlaxcala se siembran entre 60 mil y 80 mil plantas ha $^{-1}$, o bien de 20 a $25 \mathrm{~kg} \mathrm{ha}^{-1}$, según la modalidad: riego, punta de riego, humedad residual o temporal (MaríaRamírez y Rojas-Martínez, 2003). Al evaluar varias dosis de fertilización con nitrógeno, fósforo y potasio, así como densidades de población en líneas e híbridos de maíz en el noreste de México, Antonio (1998) reportó que debido a las diferencias genéticas no es posible generalizar la respuesta de líneas e híbridos a esos factores; el autor también mostró que los mejores rendimientos no coinciden con la recomendación comercial que se hace para el cultivo del maíz en esa región. En experimentos con líneas progenitoras de híbridos para Valles Altos establecidas en dos densidades de población (62 500 y 82 500 plantas ha $^{-1}$ ) para evaluar el rendimiento y calidad física de semilla, Virgen-Vargas y Arellano-Vázquez (Com. pers. $)^{1}$ encontraron diferencias entre líneas y densidades de población en rendimiento, tamaño de semilla, peso de 200 semillas y volumen de semilla; en la densidad mayor, el rendimiento aumentó de $16 \%$ a $20 \%$ y el porcentaje de semilla chica aumentó de 40 a $45 \%$, en comparación con la densidad de 62000 plantas ha $^{-1}$.

En el presente estudio se evaluó el rendimiento y la calidad física de semilla de cruzas simples progenitoras fémeninas de híbridos de maíz, sembradas en dos densidades de población en dos localidades del Edo. de Tlaxcala.

\section{MATERIALES Y MÉTODOS}

El experimento se sembró en el año de 2008 en las localidades de Espíritu Santo (19 ${ }^{\circ} 20^{\prime} 48.8^{\prime \prime}$ LN y $98^{\circ}$ $25^{\prime} 27.6^{\prime \prime} \mathrm{LO}, 2480 \mathrm{msnm}$, en temporal) y en Francisco Villa $\left(19^{\circ} 35^{\prime} 39.8^{\prime \prime}\right.$ LN y $98^{\circ} 27^{\prime} 38.3^{\prime \prime}$ LO, 2540 msnm, con riego) del Estado de Tlaxcala. Se utilizaron siete cruzas simples progenitoras hembras de tres híbridos

${ }^{1}$ Juan Virgen Vargas (2006) Producción y Tecnología de Semillas. Campo Experimental Valle de México. INIFAP experimentales y de dos híbridos comerciales de maíz para Valles Altos, de las cuales cuatro fueron formadas por el INIFAP (CS $q$ H-50, CS $\odot$ H-64 E, CS + H-66 E, CS 9 H-68 E) y tres por el Centro Internacional de Mejoramiento de Maíz y Trigo CIMMYT (CML246 X CML242, CML241 X CML243 y CML239 X CML242). El testigo fue la $\mathrm{CS} q \mathrm{H}-50$, que es la cruza femenina del híbrido 'H-50' el cual está en comercialización desde hace diez años.

El diseño de tratamientos fue un factorial completo, con base en la combinación de las siete cruzas simples con dos densidades de población (62000 y 83000 plantas ha ${ }^{-1}$ ). La parcela experimental fue de cuatro surcos de $5 \mathrm{~m}$ de longitud separados a $0.80 \mathrm{~m}$, con una distancia entre plantas de 0.20 y $0.15 \mathrm{~m}$, según la densidad; se usaron los dos surcos centrales como parcela útil. El experimento se estableció bajo un diseño de bloques completos al azar con tres repeticiones.

Los experimentos se sembraron el 20 de mayo en Espíritu Santo y el 19 de abril en Francisco Villa; se utilizaron las fórmulas de fertilización $140 \mathrm{~N}-70 \mathrm{P}-30 \mathrm{~K}$, y para el control de maleza se hicieron aplicaciones del herbicida Gesaprim ${ }^{\circledR}$ calibre 90 a dosis de $1 \mathrm{~L} \mathrm{ha}^{-1}$; además se hicieron dos aplicaciones del fertilizante foliar Grow K® (0N-40P-52K) después de la segunda labor de cultivo. También se hicieron deshierbes manuales y aplicaciones de Arrivo ${ }^{\circledR} 200$ para el control de plagas, principalmente pulgón del cogollo (Rhopalosiphium maidis Fitch).

Las variables medidas fueron: (a) Rendimiento (REND), en $\mathrm{kg} \mathrm{ha}^{-1}$ a $14 \%$ de humedad, que se calculó con la fórmula: $R E N=[P C \times \% M S x \% G x F C] / 8600$; donde $P C=$ peso de campo de mazorca, en kilogramos por parcela útil; $\% M S=$ porcentaje de materia seca, mediante la diferencia 100 menos el porcentaje de humedad obtenido del aparato Stenlite ${ }^{\circledR} ; \% G=$ porcentaje de grano, como promedio de la relación entre el peso de grano y el peso de mazorca desprovista de brácteas, de cinco mazorcas, multiplicado por 100; $F C=$ factor de corrección, obtenido al dividir $10000 \mathrm{~m}^{2}$ (1 ha) entre la superficie útil de la parcela $\left(8 \mathrm{~m}^{2}\right)$. (b) Tamaño de la semilla, mediante su clasificación en tres tamaños: grande (SG), mediana (SM) y chica $(\mathrm{SCH})$, lo cual se hizo con cribas de perforación redonda de 8,7 y $6 \mathrm{~mm}$, y los valores se reportaron en porcentajes. (c) Peso de 200 semillas (P200S), las cuales se eligieron al azar en cada muestra de semilla. (d) Peso volumétrico (PVOL), que se determinó en una báscula marca OHAUS ${ }^{\circledR}$ y se expresó en $\mathrm{kg} \mathrm{hL}^{-1}$. 
Los datos fueron analizados mediante análisis de varianza combinando las localidades. La comparación de medias fue mediante Tukey $(\alpha \leq 0.05)$. Los datos expresados en porcentaje se transformaron previamente a valores de arcoseno.

\section{RESULTADOS Y DISCUSIÓN}

En el Estado de Tlaxcala hubo una buena temporada de lluvias durante el periodo de abril a octubre del 2008, con una precipitación de $776 \mathrm{~mm}$ en Espíritu Santo y de 548 $\mathrm{mm}$ en Francisco Villa (Estaciones meteorológicas del INIFAP, San Antonio Atotonilco y Benito Juárez, Tlaxcala).

El factor genotipos (cruzas simples) afectó $(\mathrm{P} \leq 0.05)$ a todas las variables; entre localidades hubo diferencias ( $\mathrm{P}$ $\leq 0.05)$ para rendimiento y peso volumétrico; entre densidades de población sólo hubo diferencias $(\mathrm{P} \leq 0.05)$ entre semillas grandes y medianas, y en el peso de 200 semillas. En la interacción localidad x cruza simple hubo diferencias $(\mathrm{P} \leq 0.05)$ para el peso volumétrico, mientras que en la interacción localidad $\mathrm{x}$ densidad de población hubo diferencias $(\mathrm{P} \leq 0.05)$ para rendimiento y semilla grande (Cuadro 1).

En la localidad Francisco Villa se produjo el mayor rendimiento $\left(5.38 \mathrm{t} \mathrm{ha}^{-1}\right)$ pero la semilla tuvo menor peso volumétrico que en Espíritu Santo, lo que pudo deberse al riego que se aplicó en octubre para evitar efectos de sequía durante el llenado de grano. Estos rendimientos son $30 \%$ menores a los obtenidos en el 2007 en Espíritu Santo (Virgen-Vargas, Opus cit.) ${ }^{1}$, año en el que hubo una precipitación de $615 \mathrm{~mm}$ de mayo a septiembre y de $30 \mathrm{~mm}$ en octubre y noviembre (SMN, 2010). El rendimiento de semilla de las cruzas simples de los híbridos experimentales 'H-64', 'H-66' y 'H-68' superaron $(\mathrm{P} \leq 0.05)$ al de las cruzas formadas en el CIMMYT; los híbridos experimentales son progenitores de los híbridos comerciales 'H-40', 'H-50' y 'H-153'. Esta superioridad, quizás se deba a que son cruzas con mayor adaptación a las condiciones templadas de Valles Altos que las cruzas que intervienen en el ' $\mathrm{H}-40$ ' y el ' $\mathrm{H}$ 153', cuyos progenitores contienen germoplasma subtropical, y a que presentan un mejor porte de planta que la cruza del ' $\mathrm{H}-50$ '.

La calidad física de la semilla de las cruzas de los híbridos experimentales 'H-64' y 'H-66', 'H-68', medidas en peso volumétrico y peso de 200 semillas, fue mayor ( $\mathrm{P}$ $\leq 0.05$ ) que la del 'H-50' (Cuadro 2), aunque en todas las cruzas los pesos volumétricos fueron inferiores a los establecidos en la norma NMX-034 (2002). La diferencia del rendimiento entre las densidades de población no fue significativa, pero en la densidad de 83000 plantas hubo $4.0 \%$ menos de semilla grande y mediana que en la densidad de 62000 plantas (Cuadro 2).

Rojas-Martínez et al. (2009) propusieron las densidades de población de los progenitores hembra para la producción de semilla certificada en el Estado de Tlaxcala, que deben ser de 75 a 85 y de 45 a 50 mil plantas ha $^{-1}$ para riego y temporal, con las cuales se obtendría mayor tamaño y peso volumétrico de la semilla producida. En este trabajo la interacción localidad x densidades de población fue significativa para rendimiento y para el porcentaje de semilla grande (Cuadro 3). En la localidad Francisco Villa se obtuvieron 200 kg y 9 \% más de semilla grande con la densidad de 62000 plantas ha ${ }^{-1}$, en tanto que en la localidad Espíritu Santo sólo hubo diferencias en el rendimiento, pues en la densidad de 83 000 plantas ha ${ }^{-1}$ se obtuvieron $500 \mathrm{~kg}$ de semilla más que en la de 62000 plantas ha $^{-1}$.

Cuadro 1. Cuadrados medios de rendimiento, tamaño de semilla y calidad física de siete cruzas simples progenitoras de híbridos experimentales y comerciales de maíz, en dos densidades de población y en dos localidades del Edo. de Tlaxcala, Espíritu Santo y Francisco Villa. 2008.

\begin{tabular}{|c|c|c|c|c|c|c|c|}
\hline Fuente de variación & $\mathrm{gl}$ & REND & SG & SM & $\mathrm{SCH}$ & PVOL & $\mathrm{P} 200 \mathrm{~S}$ \\
\hline Localidad (L) & 1 & $3.2 *$ & 264 & 92 & 71 & $276^{* *}$ & 230 \\
\hline Cruza simple (CS) & 6 & $34.6^{* *}$ & $3521 * *$ & $361 * *$ & $1697 * *$ & $121 * *$ & $859 * *$ \\
\hline Densidad de población (DP) & 1 & 0.6 & $349^{*}$ & $219 * *$ & 77 & 1.7 & $236^{*}$ \\
\hline $\mathrm{L} \times \mathrm{CS}$ & 6 & 0.4 & 136 & 62 & 34 & $26^{*}$ & 134 \\
\hline L x DP & 1 & $2.9^{*}$ & $371^{*}$ & 59 & 119 & 3.7 & 199 \\
\hline $\mathrm{CV}(\%)$ & & 17.1 & 21.6 & 16 & 23.9 & 4.7 & 14.7 \\
\hline
\end{tabular}

*, ** Significativo a 0.05 y 0.01 de probabilidad, respectivamente; $\mathrm{CV}=$ coeficiente de variación; $\mathrm{gl}=$ grados de libertad; REND = rendimiento de grano por hectárea; $\mathrm{SG}=$ porcentaje de semilla grande; $\mathrm{SM}=$ porcentaje de semilla mediana; $\mathrm{SCH}=$ porcentaje de semilla chica; $\mathrm{PVOL}=$ peso volumétrico, en $\mathrm{kg} \mathrm{hL}^{-1} ; \mathrm{P} 200 \mathrm{~S}=$ peso de 200 semillas, en $\mathrm{g}$. 
Cuadro 2. Rendimiento de grano, tamaños y calidad física de semilla de maíz, en función de tres factores de producción. Tlaxcala, 2008

\begin{tabular}{|c|c|c|c|c|c|c|}
\hline \multirow[t]{2}{*}{ 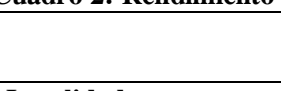 } & \multicolumn{4}{|c|}{ Tamaño de semilla } & \multirow[b]{2}{*}{ PVOL } & \multirow[b]{2}{*}{ P200S } \\
\hline & REND & SG & SM & $\mathrm{SCH}$ & & \\
\hline Francisco Villa & $5.38 \mathrm{a}$ & $39.8 \mathrm{~b}$ & $35.3 \mathrm{a}$ & $26.4 \mathrm{a}$ & $68.1 \mathrm{~b}$ & $55.3 \mathrm{a}$ \\
\hline \multicolumn{7}{|l|}{ Cruza simple } \\
\hline $\mathrm{CS}+\mathrm{H}-64 \mathrm{E}$ & $7.05 \mathrm{a}$ & $43.17 \mathrm{c}$ & $38.02 \mathrm{a}$ & $21.47 \mathrm{bc}$ & $68.58 \mathrm{~b}$ & $61.21 \mathrm{ab}$ \\
\hline $\mathrm{CS}$ ㅇ H-66 E & $6.42 \mathrm{ab}$ & $44.48 \mathrm{bc}$ & $36.53 \mathrm{ab}$ & $22.39 \mathrm{bc}$ & $69.41 \mathrm{ab}$ & $63.67 \mathrm{a}$ \\
\hline CML246 X CML242 & $5.33 \mathrm{bc}$ & $57.85 \mathrm{a}$ & $27.65 \mathrm{c}$ & $14.87 \mathrm{c}$ & $63.70 \mathrm{c}$ & $53.27 \mathrm{bc}$ \\
\hline CML239 X CML242 & $4.78 \mathrm{c}$ & $54.07 \mathrm{ab}$ & $30.12 \mathrm{bc}$ & $17.51 \mathrm{c}$ & $72.64 \mathrm{ab}$ & $48.05 \mathrm{~cd}$ \\
\hline $\mathrm{CS}+\mathrm{H}-50$ & $4.49 \mathrm{c}$ & $36.22 \mathrm{c}$ & $40.13 \mathrm{a}$ & $27.74 \mathrm{~b}$ & $70.55 \mathrm{ab}$ & $55.78 \mathrm{ac}$ \\
\hline CML241 X CML243 & $1.95 \mathrm{~d}$ & $13.66 \mathrm{~d}$ & $41.22 \mathrm{a}$ & $44.82 \mathrm{a}$ & $71.91 \mathrm{ab}$ & $38.38 \mathrm{~d}$ \\
\hline \multicolumn{7}{|l|}{ Densidad de población } \\
\hline 62 mil plantas/ha & $5.10 \mathrm{a}$ & $40.09 \mathrm{a}$ & $34.82 \mathrm{~b}$ & $26.38 \mathrm{a}$ & $70.08 \mathrm{a}$ & $63.8 \mathrm{a}$ \\
\hline
\end{tabular}

Cuadro 3. Medias de rendimiento, tamaño y peso de semilla de maíz en la interacción localidad x densidad de población. Tlaxcala, 2008.

\begin{tabular}{|c|c|c|c|c|c|c|c|}
\hline & \multirow{2}{*}{$\begin{array}{l}\text { Densidad de población } \\
\text { (miles de planta/ha) }\end{array}$} & \multicolumn{4}{|c|}{ Tamaño de semilla } & \multirow[b]{2}{*}{ PVOL } & \multirow[b]{2}{*}{ P200S } \\
\hline & & REND & SG & SM & $\mathrm{SCH}$ & & \\
\hline \multirow[t]{2}{*}{ Francisco Villa } & 62 & $5.48 \mathrm{a}$ & $43.9 \mathrm{a}$ & 32.9 & 24.3 & $68.0 \mathrm{~d}$ & 58.5 \\
\hline & 83 & $5.28 \mathrm{~b}$ & $35.6 \mathrm{~b}$ & 37.8 & 28.5 & $68.2 \mathrm{c}$ & 52.1 \\
\hline \multirow[t]{2}{*}{ Espíritu Santo } & 62 & $4.71 \mathrm{c}$ & $36.2 \mathrm{~b}$ & 36.7 & 28.5 & $72.1 \mathrm{a}$ & 52.1 \\
\hline & 83 & $5.26 \mathrm{~b}$ & $36.4 \mathrm{~b}$ & 38.2 & 28.0 & $71.4 \mathrm{~b}$ & 51.8 \\
\hline
\end{tabular}

REND $=$ rendimiento de grano, en $\mathrm{t} \mathrm{ha}^{-1} ; \mathrm{SG}=$ porcentaje de semilla grande; SM = porcentaje de semilla mediana; $\mathrm{SCH}=$ porcentaje de semilla chica; $\mathrm{PVOL}=$ peso volumétrico, en $\mathrm{kg} \mathrm{hL}^{-1} ; \mathrm{P} 200 \mathrm{~S}=$ peso de 200 semillas, en $\mathrm{g}$.

\section{CONCLUSIONES}

Las cruzas simples hembras de los híbridos experimentales 'H-68', 'H-64' y 'H-66' tuvieron mayor rendimiento y calidad de semilla que la cruza del híbrido comercial 'H-50', en dos localidades del Edo. de Tlaxcala, lo que indica la conveniencia de su producción de semilla en este estado. Entre localidades hubo una respuesta diferencial para rendimiento y porcentaje de semilla grande al incrementar la densidad de población; en Espíritu Santo fue mayor el rendimiento en la alta densidad (83 mil plantas/ha), tanto que en Francisco Villa fue mayor el rendimiento y el porcentaje de semilla grande en la baja densidad (62 mil plantas/ha).

\section{BIBLIOGRAFÍA}

Antonio M J (1998) Fertilización y densidad de población en líneas de maíz en el noreste de México. Agron. Mesoam. 9:125-130.

Beck D (2002) Seed Course. Disponible en: http://www.cimmyt.cgiar. or/Research/maize/qpm2002/Seed/Seed LN.htm (Noviembre 2008).

María-Ramírez A, I Rojas-Martínez (2003) Producción de Maíz de Temporal en el Estado de Tlaxcala. Fundación Produce Tlaxcala, INIFAP- Tlaxcala. Tlaxcala. $211 \mathrm{p}$.
Norma Mexicana NMX-FF-034-2002-SCFI-PARTE-1 (2002) Productos Alimenticios no Industrializados-para Consumo HumanoCereales-Maíz Blanco para Proceso Alcalino para Tortillas de Maíz y Productos de Maíz Nixtamalizado-Especificaciones y Métodos de Prueba. Dirección General de Normas. Secretaría de Economía. México, D. F. 18 p.

Rojas-Martínez I, J Virgen-Vargas, A Espinosa-Calderón, $\mathbf{R}$ Fernández-Sosa (2009) Tecnología para la Producción de Semilla Certificada de Maíz del Híbrido H-48 en Tlaxcala. Folleto Técnico No. 39. INIFAP. TLAXCALA. 33 p.

Servicio Meteorológico Nacional, SMN (2010) Análisis Mensual de Precipitación por Entidad Federativa. Comisión Nacional del Agua. México. Disponible en: http://www.smn.cna.gob.mx/ climatologia/precipitacion/estados/municipios/ (Febrero 2010).

Sistema de Información Agroalimentaria y Pesquera, SIAP (2009) Anuario Estadístico de la Producción Agrícola. Secretaría de Agricultura, Ganadería, Desarrollo Rural, Pesca y Alimentación. México. Disponible en: http://www.siap.sagarpa.gob .mx/ (Noviembre 2009). 